Math. Model. Nat. Phenom.

Vol. 4, No. 3, 2009, pp. 68-96

DOI: $10.1051 / \mathrm{mmnp} / 20094303$

\title{
Mathematical Modelling of Tumour Dormancy
}

\author{
K.M. Page ${ }^{1}$ \\ Department of Mathematics, UCL, London WC1E 6BT, UK
}

\begin{abstract}
Many tumours undergo periods in which they apparently do not grow but remain at a roughly constant size for extended periods. This is termed tumour dormancy. The mechanisms responsible for dormancy include failure to develop an internal blood supply, individual tumour cells exiting the cell cycle and a balance between the tumour and the immune response to it. Tumour dormancy is of considerable importance in the natural history of cancer. In many cancers, and in particular in breast cancer, recurrence can occur many years after surgery to remove the primary tumour, following a long period of occult disease. Mathematical modelling suggested that continuous growth of tumours was incompatible with data of the times of recurrence in breast cancer, suggesting that tumour dormancy was a common phenomenon. Modelling has also been applied to understanding the mechanisms responsible for dormancy, how they can be manipulated and the implications for cancer therapy. Here, the literature on mathematical modelling of tumour dormancy is reviewed. In conclusion, promising future directions for research are discussed.
\end{abstract}

Key words: cancer, dormancy, mathematical models

AMS subject classification: 92B05

\section{Introduction}

Tumour dormancy occurs in most solid and haematological human cancers [3]. It was originally defined to be a period of more than five years in which there is little change in an individual's tumour burden [1], [2]. More generally it is a period of latency in cancer progression during which time there is residual disease but the patient remains asymptomatic. Mechanisms that may be responsible for these periods of latency are cellular quiescence (G0-G1 arrest), inhibition of

\footnotetext{
${ }^{1}$ E-mail: kpage@math.ucl.ac.uk
} 
angiogenesis and immune responses to the tumour. Mathematical modelling has been applied to studying tumour dormancy in all of these contexts. Here we review the literature.

Most cancer deaths occur due to metastatic growths in vital organs. These growths will have been seeded by disseminated tumour cells. Often disseminated tumour cells are not immediately able to grow to form large tumours in their new microenvironments. Relapse commonly occurs in breast and prostate cancer decades after resection of the primary tumour [4], [5]. Early models of cancer growth proposed continuous growth profiles to describe the natural history of cancer. Data on recurrence in breast cancer in particular was difficult to reconcile with these continuous growth kinetics. This led to the suggestion of dormant phases in the tumour's history.

This article is structured as follows: first, models of recurrence in breast cancer are described, together with the statistical and modelling work that led to the proposal of tumour progression involving dormant phases. Secondly, models of tumour angiogenesis (the creation of a new tumour vasculature) are discussed. Failure to develop a supportive vasculature can lead to the arrest of growth of tumours at small sizes, at which they can derive nutrients by diffusion from outside. Models both of the process of angiogenesis and of the angiogenic switch (the change which allows tumour angiogenesis to occur) are discussed. Next, models of cellular dormancy, that is quiescence of individual tumour cells, are described. This is followed by a discussion of models of the interaction between tumours and the immune system. We sum up with a discussion of the importance of tumour dormancy, the mechanisms likely to be responsible for it, the contribution of mathematical modelling to understanding it, the implications for therapy and promising avenues for future research.

\section{Models of recurrence in breast cancer}

In breast cancer, studies have been performed for many years following up patients who have undergone therapy for primary tumours. Often therapy consists of mastectomy and sometimes additionally systemic chemotherapies and hormonal therapies and local radiotherapy. Recurrences and breast cancer-related deaths are monitored over time to assess risks in patients relative to the female population at large, reductions in risk associated with different therapies and the influence of various factors, such as patient age or size of tumour at resection. For example, the Early Breast Cancer Trialists' Collaborative Group (EBCTCG) was set up in 1984- 1985 to coordinate worldwide meta-analyses every five years "of centrally collected data on every woman in all randomised trials of the treatment of early breast cancer that had, at the time of the analysis, already been running for at least 5 years" [6].

Studies of recurrence and mortality in surgically treated breast cancer show excess mortality for 30 years after surgical therapy [7]. Mathematical models based on a cured fraction and a fraction destined to die of the cancer estimate the cured fraction to be in the range of $18-38 \%$, see for example [8]. Clearly some excess risk could be due to patients developing genuinely new tumours, because they are more genetically susceptible or because they encounter greater risk factors in their environments. However, circulating tumour cells have been found in the blood of a significant 
proportion (36\%) of breast cancer patients who had shown no clinical evidence of disease for at least seven years after mastectomy [9]. In age-matched controls there was only 1 individual in 26 who had a possible circulating tumour cell. The circulating tumour cells were short-lived in the blood (with half lives of 1-3 hours). Thus there must be some replenishing source of proliferating tumour cells. Low levels of circulating tumour cells were seen up to 22 years after mastectomy. It is therefore concluded that, in postoperative breast cancer, the cancer can remain in a dormant state for extended periods prior to recurrence. Cells from these dormant tumours clearly provided a potential source of cells for a recurrent tumour.

A great deal of effort has been pitched at statistical studies of these data on recurrence and mortality in breast cancer and some mathematical modelling (for a review see [13]) has also tried to infer plausible tumour growth profiles and other parameters which can explain the pattern of recurrences.

The favourite model of tumour growth is Gompertzian growth, in which the exponential growth rate of the tumour decays in time. It can be decribed by the following pair of equations

$$
\begin{aligned}
& \frac{d N}{d t}=K_{1} N(t) G(t) \\
& \frac{d G}{d t}=-K_{2} G(t),
\end{aligned}
$$

where $N(t)$ is the tumour volume and $K_{1}$ and $K_{2}$ are constants. $G(t)$ is a scaled tumour growth rate. The growth profile in Gompertzian growth is S-shaped, saturating at some value $N(\infty)$. These growth profiles were shown to fit very well to data on the growth of melanoma and transplantable breast carcinoma in rats [11]. However, Demicheli [16] measured the diameters of recurring tumours in 122 breast cancer patients who had undergone mastectomy. He also estimated the diameter of the tumour at the previous examination, in which the patient was deemed free of tumour. This was done by assuming the most favourable conditions to support the continuous growth model, i.e. exponential growth from a single tumour cell at the time of mastectomy. There was huge overlap between tumour diameters that had been undetectable and diameters where the tumour was detected. This argues against a model of continuous growth of the tumour, such as Gompertzian growth.

The papers of Demicheli and co-workers [14]-[16] and Karrison and co-workers [4],[17] look at the time to recurrence or death in post-operative breast cancer patients (see also [18] for a review). Demicheli et al. [14] analyse a set of data on time of treatment failure (the first clinically documented evidence of new disease) following mastectomy in 1173 patients (the Milan dataset). They plot hazard functions (estimated as the risk of failure per unit time via a particular event) for recurrence at different bodily sites. They find that the overall hazard function shows a peak at around 18 months and a second peak at around 60 months. Karrison et al. [4] plot hazard rates for time to first recurrence or death for 1547 post-mastectomy patients. They find a single- peaked hazard rate, with the peak at around 2 years post-mastectomy. Demicheli et al. [15], however, argue that Karrison's failure to see a second peak in the hazard rate was due to the fact that first recurrence and death were mixed in the data, whereas each has its own distinct time distribution. Demicheli et al. plot the hazard rate of the time to death from all causes for the Karrison data and show that it has two peaks, consistent with their own data. 
To explain their bimodal hazard rate of recurrence, Retsky, Demicheli and co-workers [11], [16] propose a mathematical model of metastatic growth. In the model, metastases progress stochastically through three stages. In the first of these, single dormant cells, which have been shed from the primary tumour, lodge in viable locations but do not proliferate. In the second stage, cells proliferate and grow into avascular micrometastases. Growth at this stage is Gompertzian, saturating at a diameter of less than $0.5 \mathrm{~mm}$. In the final stage, the metastasis becomes vascularised and grows according to Gompertzian growth, with a much larger carrying capacity, until it is detected. To start the process, the primary tumour must reach a size where it is vascularised and then single metastatic cells can be shed. Thus the model proposes two phases of dormancy which must be passed through before metastases become detectable. The model as described is capable of generating the second peak in the recurrence hazard, but not the first. This first peak was too narrow and sharp to be fitted by the model. In order to generate both peaks, the authors postulate an increase in the transition rates between states, from stage one to stage two, stage two to stage three and directly from stage one to stage three, caused by surgery. The estimated transition rates immediately after surgery are very high, suggesting that surgery may dramatically advance metastatic growth. It is suggested that this acceleration may be due to the removal of angiogenic inhibitors, which are secreted by the primary tumour, and the production of some growth-simulatory factor following surgery. The bimodal hazard rate of death of the Milan data set is compared to a dataset of 250 untreated breast cancer patients from [12]. The latter has a unimodal hazard rate of death [16]. This adds weight to the argument that surgery causes the bimodal hazard rate. An unfortunate consequence of the predictions of Demicheli et al.'s model is that the majority of tumours which relapse in the first peak have actively growing metastases following surgery. Those that relapse in the second peak do not and are likely to have dormant tumours which may not respond to chemotherapy in the months following surgery.

More recent studies fail to find the two-peaked hazard functions found in the work of Demicheli and co-workers, see for example [18], [6]. As proposed in [18], this may be due to differences between the therapies available to patients in the datasets studied by Demicheli and co-workers (who were operated on between the 1940s and 1980s) and more modern therapies.

Recent comparisons of recurrence in patients with primary tumours of different sizes suggests that the risk of metastasis and the number of disseminated tumour cells in the bone marrow scale only weakly with the size of the primary tumour [1]. Together with genomic data showing that metastatic cells from tumours often displayed significantly fewer genomic aberrations than the corresponding primary tumour cells, this suggests that tumour cells may disseminate early in the tumour history. Metastatic growth does not immediately ensue, suggesting that most disseminating cells may initially be in a state of cellular dormancy.

The existence and importance of a dormant phase in tumour growth is clearly demonstrated by models of recurrence in breast cancer. In order to address causes of dormancy, we now discuss mechanistic models of tumour development and their relation to dormancy. 


\section{Models of tumour growth}

Modelling tumour growth has a long history. A review of this enormous literature is beyond the scope of this paper and we refer the reader to [19], [20] for an introduction to this subject. We focus here on topics of specific relevance to tumour dormancy.

\section{Models of tumour angiogenesis}

One of the major causes of growth arrest of tumours leading to a dormant phase in the tumour's evolution is the absence of a blood supply within the tumour. Solid tumours can generally grow to a size of around a million cells or equivalently a diameter of $1-2 \mathrm{~mm}$ whilst obtaining nutrients, in particular oxygen, from the vasculature of the surrounding tissue. Oxygen must then diffuse from the outside of the tumour. Past a certain small size, the cells at the centre of the tumour become hypoxic and, as the tumour gets a little larger, cells at the centre begin to die. A balance between cell proliferation at the surface and death at the core gives rise to the 1-2mm constant size dormant tumours. When cells disseminate via the vasculature from a primary tumour they can exit (extravasate) into surrounding tissue seeding new small tumours. These micrometastases can form as cuffs around the vessel from which they presumably extravasated. Whilst avascular, the radii of these cuffs are restricted (in a mouse model of Lewis lung carcinoma the radius is approximately $150 \mu \mathrm{m}$ [22]). Within these cuffs, cells are proliferating, but also dying at a high rate via apoptosis.

In order to escape this dormant phase, cancer cells have to induce the generation of a new vasculature within the tumour. This process is called angiogenesis and its study in the context of cancer has been pioneered by Judah Folkman and co-workers see for example [21]-[25].

Angiogenesis depends on a balance between angiogenic promoters and inhibitors. The former are typically growth factors such as VEGF, PDGF and FGF [3], [49]-[51]. The latter include angiostatin, endostatin, thrombospondin and vasculostatin. Normal cells and tumour cells in the early dormant phase of growth typically produce an excess of angiogenic inhibitors. The hypoxic tumour cells can subsequently undergo the angiogenic switch in which the balance is shifted in favour of production of pro-angiogenic factors. There is good evidence that primary tumours can lead directly or indirectly to the secretion of angiogenic inhibitors into the blood. In Lewis lung carcinoma, angiostatin is produced and the presence of the primary tumour maintains a high apoptotic index in micrometastases, which consequently remain small [22], [23], [24]. Removal of the primary tumour leads rapidly (within five days) to the infiltration of the micrometastases by endothelial cells (commencing angiogenesis, see next paragraph) and the subsequent growth of the micrometastases. The half-life of angiostatin in the blood is 2.5 days, whereas the half-life of VEGF, which is the primary mediator of angiogenesis in this and many tumours, is only around three minutes. Thus secretion of VEGF by the primary tumour does not cause an accumulation VEGF in the blood.

Blood vessels are formed from endothelial cells which line their walls. The formation of most new blood vessels, except in embryos, involves the division and directed migration of mature differentiated endothelial cells from existing vasculature. Until recently it was thought that this was the only mechanism operative postnatally. An alternative mechanism termed vasculogenesis was 
proposed for the formation of vessels [26], [27] and subsequent evidence suggests this mechanism may also be operative in tumours (see for example [28], [29]). In vasculogenesis, neovasculature promoting factors cause a circulating population of endothelial progenitor cells (instead of fully differentiated endothelial cells) to migrate to the site of the tumour and build vessels there. These precursor cells subsequently differentiate to form the endothelial cells which line the new tumour vasculature. Some authors still call this alternative mechanism angiogenesis and it is as yet unclear how relatively important the mechanism is in tumour progression.

In order to understand angiogenic dormancy it is necessary to understand how tumour angiogenesis (or vasculogenesis) works and how the angiogenic switch is effected. Mathematical models of both of these processes are discussed below. Since the literature on mathematical models of tumour angiogenesis is very large we refer the reader to a couple of excellent, recent review articles [30], [31] for a more thorough survey.

Baum et al. [32] present a model by Anderson and Chaplain of angiogenesis in tumours. They argue that Gompertzian growth models, historically used to describe the growth of tumours, are inadequate. Dormancy is likely due to the persistence of micrometastases, that is small tumours derived from tumour cells which have passed through the blood stream and lodged at sites distant from the primary tumour. Once micrometasases reach a size of around a million cells or 1-2mm in diameter, their development is arrested awaiting a rate-limiting step, which is tumour-induced angiogenesis.

Anderson and Chaplain [33] model the growth of new blood vessels from a pre-existing vessel at one side of their spatial domain towards a tumour at the other side. The capillaries formed are lined with endothelial cells. Their model of angiogenesis has variables to describe the density of endothelial cells, $n(x, y, z, t)$, the concentration of tumour angiogenesis factors, $c(x, y, z, t)$, and the concentration of matrix angiogenesis factors like fibronectin, $f(x, y, z, t)$, which bind to the extracellular matrix and can be secreted by the endothelial cells. Tumour angiogenesis factors, such as VEGF, are secreted by the tumour and endothelial cells (ECs) can move up gradients of these factors by a process of chemotaxis. Tumour angiogenesis factors (TAFs) can diffuse in the extracellular space and decay. It is assumed that the concentration of the TAF has reached an equilibrium in the absence of endothelial cells, so that it is initially graded away from the tumour. Subsequent evolution of the TAF concentration is due only to uptake by ECs. Endothelial cells can also move by a process of haptotaxis on the extracellular matrix (ECM). This is enhanced by the presence of matrix angiogenesis factors which bind the ECM but do not diffuse. Thus it is assumed that haptotaxis occurs up gradients of fibronectin. Since plasma fibronectin may be leaked by the primary blood vessel, the authors investigate scenarios in which the fibronectin concentration is initially graded away from the primary vessel. The subsequent evolution of fibronectin concentration is modelled as only due to production and uptake by the endothelial cells. 
Their model is given by a system of partial differential equations:

$$
\begin{aligned}
\frac{\partial n}{\partial t} & =D \nabla^{2} n-\nabla\left(\frac{\chi_{0} k_{1}}{k_{1}+c} n \nabla c\right)-\nabla \cdot\left(\rho_{0} n \nabla f\right) \\
\frac{\partial f}{\partial t} & =\omega n-\mu n f \\
\frac{\partial c}{\partial t} & =-\lambda n c .
\end{aligned}
$$

where $D$ is the random motility coefficient, $\chi_{0}$ is the chemotactic response parameter and $\rho_{0}$ is the haptotactic response parameter of the ECs, $\lambda$ is the rate of TAF uptake by the ECs, $\omega$ is the rate of production of fibronectin by the ECs and $\mu$ is the rate of fibronectin uptake by the ECs. ECs are desensitised to chemotaxis at high TAF concentration so that the chemotaxis response function is given by a receptor kinetic law $\chi(c)=\chi_{0} k_{1} /\left(k_{1}+c\right)$. Equations (4.1) are subject to zero flux boundary conditions.

Numerical simulations of this continuum model with three initial peaks of EC concentration at the parent vessel end of the two-dimensional spatial domain, show that the areas of cell density (corresponding to vessel tips) move directly across the domain towards the tumour and do not fuse in the absence of haptotaxis. When haptotaxis is incorporated, lateral movement is possible, the "vessels" fuse and movement across the domain is slower. If the chemotactic response at high TAF concentration declines sufficiently then endothelial cells may never reach the tumour.

In order to model the movement of individual cells at the tip of forming blood vessels rather than an endothelial cell density, the authors discretize the model of equation (4.1), using Euler finite differences, to give a discrete biased random walk. This determines probabilities for movement of the leading cell of a vessel (sprout) tip to points on a square lattice in the von Neumann neighbourhood of its current location. TAF and fibronectin concentrations are deterministically updated according to the appropriate discretized rules, which depend on the presence/ absence of EC tip cells at the spatial location.

In addition to these equations which guide the movement of the leading cell in a forming blood vessel, other cells are assumed simply to follow that cell. Rules are then applied for the branching of vessels and their fusion to form a loop (anastomosis). Behind the tip of the blood vessel sprout, cells can also proliferate, which is implemented as elongation of the sprout.

In the discrete model, haptotaxis is once again necessary for generating sufficient lateral movement of vessels to obtain a realistic network structure. The rules for branching assume that it is more likely at high TAF concentration and the model could thus reproduce the formation of a "brush border" (highly branched vessel region) near to the tumour. Cell proliferation in the vessel sprout is necessary to achieve complete migration of vessels across the domain to connect with the tumour, given the retarding effect of a desensitising chemotactic response.

The authors attempt to estimate parameters from data in so far as possible. Overall they find that capillary networks form on a timescale between 10 and 21 days when the distance from the parent vessel to the tumour was $2 \mathrm{~mm}$. This is consistent with experimental data for angiogenesis in the mouse cornea in response to a tumour implant, in which an average distance was 1-2mm [34]. 
It appears then that latent periods of tumour growth of the order of a month may be realistic for avascular micrometastases lodged close to existing vasculature to develop their own blood supply in order to expand further. Longer periods of latency may be due to the micrometastases initially not producing sufficient pro-angiogenic factors to effect the "angiogenic switch" (see below) and stimulate new vessel formation.

For some years, mathematical models such as the one described above by Anderson and Chaplain, focussed on the proliferation and migration of endothelial cells through the extracellular matrix towards the tumour. More recently several authors have considered blood flow in the developing vessel network and its influence on vessel morphology (see for example [35], [36], [37], [38]). The morphology of tumour vasculature and the blood flow therein can have implications for the delivery of nutrients and of chemotherapeutic drugs to the tumour.

Pries and co-workers [39]-[41] have developed models of structural adaptation of microvasculature in response to blood flow. They provide a phenomenological model of blood viscosity as a function of vessel radius and haematocrit (volume fraction of red blood cells, which constitute the majority of the solid phase of the blood). Poiseuille's law can be used to relate the flow in a vessel to the pressure drop and conservation of mass can be applied at each vessel junction. This yields a linear system of equations for the junction pressures, which can be solved to yield the flows in each vessel. The wall shear stress can be calculated from the flow rate, viscosity and radius of the vessel. Increasing the wall shear stress tends to widen vessels, which reduces the wall shear stress. Other components affecting the vessel radius derive from intravascular pressure, from a low-flow haematocrit-related "metabolic" term (see [40] for details) and from a natural shrinking tendency. Updating the vessel radii in response to these mathematically described factors leads to dynamic remodelling of the vasculature when blood flows.

Chaplain et al. [31] employ this framework for modelling structural adaptation of microvasculature to modify and extend the tumour angiogenesis model of [33]. In the model of [31], degradation of fibronectin depends on the concentration of a matrix degrading enzyme, which is produced by the cancers cells and which diffuses, rather than depending directly on the cancer cell density. The model otherwise operates as described in [33] until looped vessels form. Blood can then flow in the vessels modifying their radii as described in [39]-[41]. In addition, once blood flow is established, the probability of vessel branching is assumed to increase with wall shear stress. Vessels are allowed to branch, with probability increasing with TAF concentration and with wall shear stress, provided they are aged 4-8 days. This age restriction means that they are sufficiently mature to branch, but not to have acquired a basal lamina which would prevent branching. The model predicts that reduced haptotaxis leads to reduced lateral vessel migration and to reduced wall shear-induced branching. This allows increased access of a therapeutic drug, which is added to the blood, but also allows increased nutrient access to the tumour. A similar effect is observed if the pressure in the inlet parent vessel is reduced. Although this models tumour angiogenesis, the tumour is treated as a static entity.

Other authors have developed models of vascular adaptation and tumour growth. In [35], blood flow distribution is first calculated in an adapting vascular network, in a similar manner to that described above for [31]. In addition, the haematocrit is modelled as dividing unequally between 
the downstream branches at a vessel bifurcation - more haematocrit enters the faster branch. Then oxygen is assumed to cross vessel walls with a flux proportional to the concentration drop across the wall (the blood oxygen concentration is equivalent to the haematocrit). Oxygen diffuses in the extracellular space and is used up by cells, equilibrating rapidly for a given distribution of cells. A hybrid cellular automaton approach is used to describe the discrete cells and vessels and the continuous oxygen concentration. Tumour and normal cells proliferate, die or become quiescent, depending on whether the local oxygen concentration exceeds prescribed thresholds, with proliferation occurring only if there is adjacent space for the daughter cells. The growth of the tumour and normal cell populations is compared with growth according to the same rules but with a regular lattice of identical vessels with the same amount of oxygen in each vessel. In the inhomogeneous case, the number of cancer cells saturates at a much lower level than the homogeneous case, where they ultimately cover the majority of the domain. Angiogenesis is not considered in this paper - vessel adaptation precedes tumour growth.

A rather different approach to modelling angiogenesis focuses on the dynamics of signalling in the VEGF-Bcl-2-CXCL8 pathway, which enhances endothelial cell survival and proliferation and exerts a chemoattractant effect, causing endothelial cells to align in vessels [42]. The mathematical model attempts to reproduce experiments in which polymer matrices containing oral squamous carcinoma cells and human endothelial cells are implanted into mice. The vessels in the growing tumours form de novo from the human endothelial cells rather than from sprouting of the existing mouse vasculature. VEGF is produced by hypoxic tumour cells and it upregulates the anti-apoptotic Bcl-2 in endothelial cells, which in turn upregulates interleukin-8 (CXCL8). VEGF exerts its effect by dimerising its receptor on the endothelial cells' surfaces. Interleukin-8 must bind its receptor to exert an effect. Both dimerised VEGF receptors and bound interleukin-8 receptors increase the proliferation rate of endothelial cells and enhance the rate at which endothelial cells form vessels. In addition, it is assumed that the death rates of endothelial cells and vessels decrease with increasing Bcl-2 density per cell, which is assumed to be proportional to the cellular density of dimerised VEGF receptors.

The model focuses on an accurate description of the kinetics of VEGF receptor dimerisation and interleukin- 8 binding. The binding reactions are modelled using ordinary differential equations with mass action kinetics to describe the concentrations of free ligands, receptors, bound ligands and the VEGF- VEGF receptor dimer. Free VEGF is produced in proportion to tumour cell density, when microvessel density is below a threshold (i.e. when tumour cells are hypoxic). Free interleukin-8 is produced in proportion to endothelial cell density at a basal rate plus a rate proportional to the density of VEGF- VEGF receptor dimers per endothelial cell. There is no spatial component to the model: tumour cell density, endothelial cell density and microvessel density are modelled as functions of time. The tumour cell density satisfies an ordinary differential equation. Its proliferation rate increases and its death rate decreases with oxygen concentration, which increases with microvessel density. It is assumed there is a time delay between endothelial cells receiving a signal from activated cell surface receptors and differentiating to form mature blood-bearing microvessels. Thus the differential equations for the microvessel density and the free endothelial cell density incorporate a time delay.

In simulations, the microvessel density is initially zero and does not begin to increase for five 
days, which is the time delay between endothelial cells receiving a signal and differentiating to form mature blood-bearing vessels. The tumour cells are initially hypoxic and thus the tumour cell density decreases. When microvessel density begins to grow, so does tumour cell density. Tumour cell density ultimately saturates at its maximal value. Parameter sensitivity analysis is used to investigate the dependence of the maximal steady state densities of tumour cells and vessels and the times taken to reach these on the rate at which tumour cells become necrotic at low oxygen concentration and on the tumour cells' ability to produce VEGF. The maximal tumour cell density decreases with increasing rate of necrosis at low oxygen and the time taken to reach steady state increases. However, the maximal microvessel density only decreases marginally. Thus, if tumour cells are more sensitive to local oxygen concentration, the tumours have much lower cell densities, but they remain highly vascularised. As VEGF production ability is decreased, the maximal tumour cell and vessel densities decrease marginally. The time taken to reach steady state, however, increases significantly. Thus, a reduction in the ability to produce VEGF results in a slowing of vessel formation, but is not enough to affect the steady state vessel/ tumour density.

The authors investigate two in silico therapies: i) inhibiting the production of interleukin- 8 by endothelial cells and ii) blocking VEGF-mediated upregulation of Bcl-2 by endothelial cells. A reduction in the production rate of interleukin- 8 does not affect the maximal levels of tumour cell and vessel density. However, when applied immediately upon implantation of the tumour and endothelial cells, it does slow vessel growth and tumour development. Therapy resulting in a $41.1 \%$ decrease in interleukin- 8 production, for example, results in a tumour which is $32 \%$ smaller and contains $50 \%$ fewer vessels after 21 days, matching experimental data. The anti-Bcl- 2 therapy is implemented by increasing the death rates of endothelial cells and vessels and by reducing the production rate of VEGF. Simulations can mimic the results of in vitro experiments in the absence of tumour cells, in which endothelial cells are exposed to a fixed concentration of VEGF, with and without anti-Bcl-2 therapy. Anti-Bcl-2 therapy is introduced at day five and results in a reduction in microvessel density. Good agreement with experiments is found if the therapy is efficient in blocking the effect of Bcl-2. In simulations with tumour cells included and with $100 \%$ blockage of Bcl-2, the maximal tumour cell density is reduced by around $30 \%$ and the microvessel density by around $60 \%$. The growth is also retarded, taking about twice as long to reach steady state. It is shown that a significant effect on tumour and microvessel growth depends upon the level of blockage of Bcl-2 upregulation exceeding a threshold. Further simulations address the effect of each type of therapy administered when the tumour and its microvasculature have already reached steady state. It is shown that anti-interleukin-8 therapy has little or no effect on the tumour and its vasculature when applied at this late stage. By contrast, anti-Bcl-2 therapy causes the tumour and vasculature to regress and then to stabilise at a lower steady state.

\section{Models of the angiogenic switch}

The initiation of angiogenesis is determined by shift in the balance between angiogenic activators (such as VEGF) and angiogenic inhibitors (such as endostatin). This balance between pro- and anti-angiogenic factors is termed the angiogenic balance [50], [51]. In the multistep progression 
of a cancer, mutations occur, in genes such as p53, which upregulate angiogenic activators and/or suppress inhibitors (wildtype p53 suppresses VEGFA). Hypoxia also stimulates tumour cells to produce pro-angiogenic factors, thus initiating angiogenesis.

Plank et al. [47] model the movement of endothelial cell density in response to a flux of the pro- angiogenic factor VEGF from a tumour at one side of a domain. They use a reinforced random walk framework [48] and their model incorporates chemotaxis and diffusion of ECs and proliferation of a subpopulation of immature ECs. It is assumed that immature endothelial cells produce an angiopoietin, Ang-2, which causes them to proliferate if sufficient VEGF is available or to die if not, and increases the migration rate of all ECs, thus destabilising vessels. Another angiopoietin, Ang-1, limits diffusion and proliferation/ death of ECs. It is assumed to be upregulated by peri-endothelial support cells, that is it is produced everywhere at a very low basal rate but at a higher rate near mature ECs. Its effect is to stabilise vessels. VEGF is assumed to cause vessel maturation. Solving the random walk equations leads to a wave of EC density crossing the domain, with immature cells at the front of the wave, having high Ang-2 concentration, and mature cells following, at high Ang-1 concentration. Simulations of the model show that, in the absence of Ang-2, ECs do not reach the tumour. If VEGF is present without Ang-2, even for an hour, ECs may die, causing vessel regression, and be unable to re-grow, since the low density of immature cells prevents the production of sufficient Ang-2 to allow proliferation.

These authors model the angiogenic switch within the EC compartment as the decision to be active (proliferate/ die/ migrate) at the capillary tips versus the decision to be quiescent away from there. However the angiogenic switch is more typically viewed as a change of phenotype of tumour cells which initiates the process of new vessel formation [49].

Wodarz et al. [43], [44] present a model of multifocal tumour growth dependent on the competing effects of an angiogenesis promoter and an inhibitor. (Their model is in fact conceived more generally to apply to any tumour promoter and inhibitor.) Cancer cells with population $C$ produce both promoter and inhibitor with populations $P$ and $I$ respectively. The growth rate of the cancer cell population then increases with the local blood supply which is an increasing function of $P$ and a decreasing function of $I$. It is assumed that cancer cells migrate and that the inhibitor diffuses, but that spread of the promoter is negligible. The model is described by the following system of partial differential equations on a one-dimensional domain of length $L$ :

$$
\begin{aligned}
\frac{\partial C}{\partial t} & =\left(\frac{r C}{\epsilon C+1}\right)\left(\frac{P}{I+1}\right)-\delta C+D_{C} \frac{\partial^{2} C}{\partial x^{2}}, \\
\frac{\partial P}{\partial t} & =a_{P} C-b_{P} P, \\
\frac{\partial I}{\partial t} & =a_{I} C-b_{I} I+D_{I} \frac{\partial^{2} I}{\partial x^{2}}, 0 \leq x \leq L .
\end{aligned}
$$

Here $a_{P}$ and $a_{I}$ are the production rates of the promoter and inhibitor respectively by the tumour cells and $b_{P}, b_{I}$ their decay rates. $r$ is the intrinsic growth rate of the cancer cells and $\epsilon$ a parameter describing growth saturation with increasing tumour size. $\delta$ is the death rate of the cancer cells and $D_{C}$ their migration rate. $D_{I}$ is the diffusion rate of the inhibitor. It is further assumed that the 
dynamics of the production and decay of the promoter are sufficiently rapid such that the promoter is in quasi-equilibrium with the cancer cell population (i.e. $P=a_{P} C / b_{P}$ ). Substituting this into the system of partial differential equations (PDEs) yields a system of two coupled PDEs for $C$ and $I$. These are assumed to satisfy zero flux boundary conditions.

The equations have a stable zero steady state $C=0, I=0$. In addition for some values of the parameters they have a positive spatially homogeneous steady state with $C>0, I>0$, which is stable to spatially homogeneous perturbations, but may be unstable to inhomogeneous perturbations of a range of spatial frequencies. In other words, the equations exhibit a Turing bifurcation and so for certain values of the parameters the longterm steady states are spatially periodic and consist of peaks of cancer cell number. The parameter $a_{I}$ is used as a bifurcation parameter. If it is very large, then the system collapses to the zero steady state (realistically it is proposed that there remains only a small subpopulation of cancer cells which can survive independent of the promoter). If $a_{I}$ is decreased, the cancer cells can grow, but remain confined to a single peak in the middle of the domain, since secretion of the strong inhibitor prevents growth elsewhere. As $a_{I}$ is further decreased, it can only prevent cancer cell growth in the more immediate periphery of a peak of cancer cells and multiple tumour foci can form within the domain. The distance between lesions decreases as the strength of inhibition decreases. Finally, when $a_{I}$ is sufficiently small, tumour cells can invade the entire space and the spatially homogeneous positive steady state is stable.

During tumour progression, cancer cells can evolve to produce more angiogenic promoters and a lesser quantity of angiogenic inhibitors. Provided these angiogenic cancer cell mutants can invade to a reasonable proportion the cancer cell population, then small unifocal tumours can split into multiple foci, or if the initial mutation sufficiently shifts the balance from inhibitor production to promoter production then a unifocal tumour can directly invade the whole spatial domain.

In a somewhat similar approach to the one of [43], Ramanujan et al. [45] consider a spherical tumour of radius $R$. The tumour is considered to be at steady state so that $R$ does not change. A generic proangiogenic factor and a generic antiangiogenic factor are produced, decay and diffuse. The production, decay and diffusion rates are constant within the tumour and outside the tumour in the surrounding normal tissue, but these rates differ between the tumour and normal tissue. The consequent reaction diffusion equations describing the pro- and anti-angiogenic factor concentrations are assumed to be in steady state. Boundary conditions are imposed that ensure that the concentrations are finite at $r=0$ (in spherical polar coordinates) and as $r$ tends to infinity and that concentrations and fluxes match at $r=R$ (the tumour boundary). Exact, analytical solutions are found for the concentrations as functions of $r$. The concentrations are normalised such that they take value 1 as $r$ tends to infinity. It is assumed that wherever the normalised proangiogenic factor concentration exceeds the normalised antiangiogenic factor concentration, angiogenesis is stimulated. Where the reverse is true, angiogenesis is suppressed. For baseline values of the parameters, the spatial concentration profiles show a region of angiogenesis suppression at the centre of the tumour (considered to correspond to a region of focal necrosis) and a region of angiogenesis stimulation at the outside of the tumour and in the peri-tumoural space. Finally far from the tumour the angiogenesis stimulation and inhibition balance. Parameter sensitivity analysis shows 
that as parameters such as the anti-angiogenic factor production rate outside the tumour is varied, the system can exhibit three behaviours. For very low rates, there can be global angiogenesis; for very high rates, global suppression (corresponding to tumour dormancy) and for medium rates focal suppression, as described above. The profiles of concentration ratios for focal suppression qualitatively match experimental data on tumour perfusion in rat mammary tumours.

Ramanujan et al [45] additionally describe a dual-site model of the angiogenic balance in a primary tumour and a metastatic site. The pro- and anti-angiogenic factors can circulate in the blood taking a specified time (estimated as one minute) to perform a full circulation. The primary tumour provides a source of these factors, so that it is assumed that an amount proportional to the primary tumour concentration enters the blood on each circulation. The concentrations decay exponentially in the blood and it is assumed that the system is at steady state, so that the decay occurring in one full circulation is equal to the amount added from the primary tumour. The concentrations at the metastatic site are less than those at the primary tumour by an exponential factor determined by the time taken for the blood to flow from primary tumour to metastatic site. Antiangiogenic factors have half-lives of the order of hours in the blood, whereas pro-angiogenic factors have half-lives of the order of minutes. The primary tumour concentrations are approximated by averages across the tumour volume (determined from the model described in the previous paragraph). Baseline parameter values yield a ratio of primary tumour concentrations of pro-angiogenic factor to anti-angiogenic factor which is well below the critical value for stimulation of angiogenesis at the metastatic site for a range of values of the ratio of release efficiencies and times of delivery of factors from primary to metastatic site. This means that angiogenesis suppression by the primary tumour is typical at the metastatic site (largely because of the difference in half-lives of angiogenesis inhibitors and promoters). Parameter sensitivity analyses showed that larger primary tumours and longer circulation times produced a greater suppressive influence.

Wodarz and Krakauer [46] model tumour progression from healthy cells via a mutation which gives rise to tumour cells which are not capable of angiogenesis and thus have small carrying capacity to a second mutation which gives rise to angiogenic tumour cells. The cell populations are modelled by ordinary differential equations. All cell populations have logistic growth kinetics. In addition, the angiogenic switch is inhibited by the presence of angiogenic inhibitors. The death rate of the angiogenic tumour cell population is thus modelled as increasing with the number of normal and nonangiogenic tumour cells. The death rate decreases (for suitable values of the parameters) with increasing angiogenic tumour cell number, corresponding to the production of angiogenesis promoters. The authors consider whether the angiogenic tumour cell type can invade. Depending on the strength of inhibition of the angiogenic tumour cells by the normal and nonangiogenic tumour cell types, the model shows three behaviours. If inhibition is low, then the angiogenic tumour cell type invades (the nonzero steady state only is stable). If the inhibition takes an intermediate value, both the zero and nonzero steady states of the angiogenic tumour cell type are stable; the angiogenic tumour cell type can invade only if its initial value is sufficiently large. If the inhibition is high then only the zero steady state is stable and the angiogenic tumour cell type cannot invade. In the intermediate regime, invasion of the angiogenic tumour cells depends on the mutation rate from non-angiogenic cells being large enough to give a sufficient initial cell number 
to overcome inhibition. The authors claim that this requirement for a high mutation rate could indicate the necessity for genomic instability in order for angiogenic tumour cells to invade. Thus inhibition of angiogenesis acts as a host defence. Genomic instability, which confers a selective disadvantage on cells, can allow the evolution of angiogenic tumour cells by increasing the founder population of these cells, enabling them to overcome the inhibition.

The process of tumour angiogenesis and its initiation via the angiogenic switch have been relatively well explored through mathematical models. Models of another mechanism of dormancy - quiescence at the cellular level - are now discussed.

\section{Models of cellular quiescence in cancer}

As mentioned above, models of recurrence in breast cancer [11], [16] suggest that disseminated tumour cells pass through a phase of 'cellular dormancy', in which they lodge singly in secondary organs and exist in $G_{0} / G_{1}$ arrest. Experimental evidence exists for solitary dormant cancer cells remaining for prolonged periods [54], [52], [53], [3], [18]. Molecules such as p21, p27, Myc and uPAR, which are involved in control of the cell cycle are implicated in cellular dormancy [3], [55], [56]. On the one hand, it is proposed that this form of dormancy may result from failure of the tumour cells to interpret signals from their new microenvironments, leading to stress signalling and quiescence [3]. In addition, there is some evidence that primary tumours may promote quiescence of solitary metastatic tumour cells [57]. On the other hand, it is proposed that solitary dormant tumour cells may be cancer stem cells [58], [59].

Whilst there is experimental evidence for cancer cells existing in a prolonged quiescent state (see e.g. [52], [53]), relatively little mathematical modelling work addresses the individual tumour cell's decision to exit the cell cycle (but see [60]). A range of multiscale models of tumour growth do incorporate cell cycle models at the cellular level (see e.g. [61]-[64]). These track intracellular levels of cyclins, cyclin dependent kinases (CDKs) and their inhibitors. The cyclins and CDKs undergo oscillations, leading to progression through the cell cycle, but signalling can cause an exit from the parameter regime of oscillation, indicative of quiescence. This then influences the dynamics of the tumour cell population.

The models of Alarcon and co-workers [61]-[63] link submodels of cancer growth at different spatial scales. At the subcellular level, progress though the cell cycle and the production of VEGF are modelled. At the cellular level, competition between normal and cancer cells for oxygen is considered. At the tissue level, transport of oxygen and VEGF, blood flow and vascular adaptation are modelled. Coupling between the scales is achieved, for example, because the local oxygen concentration, determined at the tissue scale, influences progression though the cell cycle and VEGF production at the subcellular level. The multiscale model of Ribba and co-workers [64] differs in the type of intracellular model of cell cycle progression. Rather than differential equation models of the levels of cyclins and CDKs and their inhibitors, these authors employ a Boolean model of the gene network with logical functions determining the discrete time updating of each gene. Cells become quiescent if either SMAD is activated, due to low oxygen concentration, or APC is activated, due to cell overcrowding. 
The $G_{1}-S$ transition, through which cells must pass in order to proliferate, has been modelled for general mammalian cells [65]. The model involves binding of a cyclin to a CDK, causing phosphorylation of a pocket protein, which leads to the release of a transcription factor involved in $\mathrm{S}$ phase activities. The cyclin-CDK complexes can be negatively regulated by cyclin dependent kinase inhibitors, which include p21 and p27, mentioned above as controlling cellular dormancy. A system of ordinary differential equations is derived. The dynamics can be oscillatory (corresponding to cell proliferation) or can tend to a fixed point (corresponding to quiescence), depending on parameter values. For example, when the concentration of cyclin dependent kinase inhibitor is below a threshold cells proliferate. Above the threshold cells enter cell cycle arrest.

More common are structured population models of tumour dynamics, in which cells are assumed to transition between proliferative and quiescent states at random with exponential waiting times. The evolution of the average numbers of proliferating and quiescent cells can then be described by ordinary differential equations. Gyllenberg and Webb [66] show that allowing the transition rate from proliferation to quiescence to increase and the reverse transition rate to decrease with tumour size means that, as the tumour grew, its growth fraction decreases and so the tumour can show a Gompertzian growth profile.

As another example, Komarova and Wodarz [67] model the influence of cellular quiescence on therapy in chronic myeloid leukaemia. Treatment with Imatinib is assumed to raise the death rate of proliferating cells, whereas quiescent cells are assumed not to die. The simplest form of the model has average numbers of proliferating and quiescent cells, $x(t)$ and $y(t)$, satisfying

$$
\begin{aligned}
& \frac{d x}{d t}=(l-d-\alpha) x+\beta y, \\
& \frac{d y}{d t}=\alpha x-\beta y,
\end{aligned}
$$

where $l$ is the cell division rate, $d$ the death rate of proliferating cells, $\alpha$ the rate of entry to quiescence and $\beta$ the rate of cell-cycle re-entry. The total cell population can undergo a biphasic decline in response to treatment, as a result of quiescence, which appears to agree with clinical data. A stochastic model, in which drug resistance mutations can occur at cell division, is used to show that the probability of treatment failure with a single drug is independent of the rates of entry and exit from quiescence. The probability of treatment failure with more than one drug, however, increases with quiescence (i.e. with increased $\alpha$ or reduced $\beta$ ). In both cases, resistance mutants leading to failure are likely to arise in the tumour growth phase prior to treatment rather than during treatment despite the fact that quiescence prolongs this treatment phase. Limited attention has been directed at modelling the decision of tumour cells to become quiescent, which is an important component of tumour dormancy. The rather more extensive literature on the interaction between tumours and the immune system is now discussed.

\section{Models of the immune response to tumours}

Perception of the importance of the immune system in controlling the growth of cancers has had a turbulent history. Spontaneously arising tumours are essentially host cells, which therefore may 
evade attack by the immune system. Nevertheless tumour cells do express antigens on their cell surfaces which are either absent on normal cells (tumour-specific antigens) or present at much lower levels (tumour-associated antigens). These supply potential targets for immune recognition. The immunosurveillance hypothesis (for a review see [70]) says that small tumours arise frequently in the body, but are eliminated by the immune system. Studies in mice in the 1970s and 80s [71], [72] showed that tumour dormancy could result when pre-immunised mice were challenged with tumour cells from genetically identical mice. These studies demonstrated the potential importance of the immune system in controlling cancer growth. However, the immunosurveillance hypothesis became unfashionable when it was apparently shown that relatively few types of cancers showed significantly increased incidences in nude mice (which have severely compromised immune systems) over wildtype mice [70] and references therein. The exception was the increased incidence of virally induced tumours, where immunosuppression was likely to be more relevant in reducing the immune system's role in preventing viral infection leading to oncogenic transformation, rather than in stemming tumour growth. Recent studies have given rise to a resurgence of interest in the immune system's involvement in attacking cancers. These show that the presence of a strong immune response to cancers, such as melanoma, colorectal and ovarian cancers, is correlated with a favourable prognosis [73]-[75]. Other studies show that primary tumours induced by chemical carcinogens in mice [76] can be controlled by the adaptive immune system (dependent on CD4+ $\mathrm{T}$ cells, CD8+ T cells and interferon- $\gamma$ ) to form small stable masses. This equilibrium involves reduced proliferation of cancer cells and increased apoptosis. Tumour cells which escape immunecontrolled equilibrium show reduced immunogenicity (measured by the efficiency of growth when transplanted into naive mice) and so it seems that the immune response to the tumours results in immuno-editing of the tumour cells.

As mentioned above, tumour dormancy can result from the interaction of the tumour with the immune system of the host, see e.g. [71], [72], [68], [69]. For example, in the mouse BCL1 lymphoma, active immunization with the cancer cell idiotype can prevent this transplantable, aggressive tumour from rapid growth. Dormant tumours of around a million cells in size can remain for up to two years (a long time relative to the mouse lifespan) [69]. Ordinary differential equation models of the interaction between the tumour and anti-idiotypic antibody are presented in [77]. The cellular immune response to the tumour is also known to be important and ordinary differential equation models of the T cell response to the tumour are given in [78], [79].

In general, a huge number of models of the interaction between tumour and immune system have been published. For reviews, see for example [80], [81].

Chapter 4 of [80], by Bellomo, Preziosi and Forni, describes an interesting class of models, called the cellular kinetic theory. A key feature of these models is that cells are ascribed a functional activation state. If, for example, the population considered consists of tumour cells, then the activation state of a cell could describe its tendency to proliferate. The state is described by a variable $u \in[-1,1]$. For the purposes of simulation, the permitted values of $\mathrm{u}$ are discretized. The density of cells of type $i$ with activation state $u$ is given by $N_{i}(t, u)$. Cells interact with other cells at some rate. These interactions can cause cells to switch activation states. The outcome of an interaction depends (probabilistically) on the type and state of both cells involved. Interactions may also result in proliferation or destruction of either cell. 
An example model is set up in which the various transition, proliferation and destruction rates are specified. There are three cell types: tumour cells, immune cells and host environmental cells. The latter are assumed to be present at a constant level and all to be fully active. Proliferation of tumour cells occurs when active tumour cells $(u>0)$ interact with suppressed immune cells $(w<0)$ or host environmental cells, and the rate is proportional to the activity of the tumour cells and to minus the activity of the immune cells. Proliferation of immune cells occurs only in through interaction with active tumour cells. Active immune cells are also supplied by the bone marrow. Destruction of active (proliferating) tumour cells occurs only in interaction with active immune cells. Destruction of immune cells occurs at a constant rate. When cells interact, they can also undergo transitions, in which the final state is distributed around a most probable value. The only interactions leading to changes of state are between tumour cells and immune cells. Active tumour cells cause immune cells to decrease their activity, whereas dormant tumour cells exert no effect. Suppressed immune cells cause tumour cells to increase their activity, whereas active immune cells cause active tumour cells to reduce their activity, but leave dormant tumour cells unchanged.

Numerical simulations of the system, varying key parameters, such as the rate of destruction of tumour cells by the immune system, show various behaviours: i) the tumour can grow rapidly and indefinitely, ii) a small tumour can grow and shrink for an extended period before growing rapidly and indefinitely, iii) after initial oscillations, the immune system can control the tumour to a constant small size or iv) the tumour can be eliminated. In the limits of total suppression or total activation of the immune system, the integro- differential equations for the densities of cell types of given activation level can be replaced by simple ordinary differential equations for the total densities of cell types.

This approach to modelling cellular interactions allows the study of heterogeneity within cell populations. Functional traits such as the tendency to proliferate in tumour cells can evolve, according to interactions with other cell types or due to genetic changes involved in tumour progression. The approach is relevant to the early stages of tumour growth, when aggregation of the tumour as a solid mass is negligible and so interactions occur randomly at the cellular level.

An elegantly simple model of the interaction between a tumour and the immune system is given in [82]. There are three variables given by immune effector cell density at the tumour site, tumour cell density and interleukin-2 (Il-2) concentration. Il-2 is the main cytokine responsible for lymphocyte activation, growth and differentiation and is commonly used in adoptive cellular immunotherapy. This therapy involves taking leukocytes from a patient's peripheral blood or from a tumour, culturing them with high concentrations of Il-2 and injecting them back into the tumour site. The aim of the model was to investigate the role of cytokines (in particular Il-2) in tumour dynamics. The model variables satisfy ordinary differential equations with terms to account for tumour growth (a logistic term), tumour cell kill by the effector cells, recruitment of effector cells by the tumour, Il-2-induced proliferation of effector cells, death of effector cells, addition of effector cells by adoptive cellular immunotherapy, production of Il-2 by interaction by effector cells interacting with tumour cells, degradation of Il-2 and therapeutic addition of Il-2. The rate at which tumour cells recruit effector cells is given by the tumour antigenicity, $c$.

In the absence of therapy, the authors investigated the tumour-immune dynamics as the param- 
eter $c$ varied. For very small values of the antigenicity, a single stable (plus two unstable) steady state exists and the tumour burden converges to a very large value. For intermediate values of antigenicity, the stable steady state and one of the unstable steady states are lost and there exists a stable limit cycle. Thus the tumour burden, the effector cell population and the Il-2 concentration oscillate. As the antigenicity increases, the amplitude of the oscillations and their period decreases. Towards the upper end of the interval in $c$, the tumour burden is very low. At high antigenicity, a stable steady state with very low tumour burden becomes stable. The oscillations exhibited in the intermediate antigenicity range are considered to explain periods of dormancy followed by recurrence of the tumour.

Therapy is modelled by adding effector cells to the tumour site at some constant rate (adoptive cellular immunotherapy) and/or adding Il-2 to the tumour site at a constant rate. When immune effector cells are added at rate $s_{1}$, then if $s_{1}$ is below a critical threshold, the same behaviours as in the no therapy regime are seen. When $s_{1}$ is nonzero but subthreshold, the critical values for $c$ for the different behaviours decrease with $s_{1}$. Above the critical threshold in $s_{1}$, there are two behaviours depending on the value of $c$. For intermediate or high antigenicity (or any antigenicity if $s_{1}$ is sufficiently large), the tumour is cleared and there are effector cells, but no Il-2 present at steady state. For low antigenicity and $s_{1}$ not too far above the threshold, the system is bistable and converges either to the state in which the tumour is cleared or the high tumour steady state, depending on the initial conditions.

When cytokine therapy is modelled, Il-2 is added to the site of the tumour at rate $s_{2}$. When $s_{2}$ is below a critical threshold, then as for adoptive cellular therapy, the same behaviours as for the no therapy regime are seen, albeit with critical values for c which decrease with $s_{2}$. If $s_{2}$ is above the critical threshold, the tumour is cleared, Il-2 concentration converges to a constant level and the effector cell population grows indefinitely. This means that Il-2 therapy alone is predicted not to have a satisfactory outcome, since the only regime in which the tumour is cleared has indefinite growth of the immune effector cell population. This indefinite growth is likely to be hazardous to the patient.

Overall it is predicted that sufficiently strong adoptive cellular immunotherapy can lead to clearance of the tumour in a satisfactory manner. Simulations with both $s_{1}$ and $s_{2}$ nonzero show that Il-2 can augment the adoptive cellular immunotherapy. Il-2 therapy alone however is unlikely to be effective.

De Pillis et al. [83] use a system of ordinary differential equations to model the interaction between tumour cells, the nonspecific immune system (natural killer cells) and the specific immune system (CD8+ T cells). Their model also considers a population of circulating lymphocytes, indicative of general immune health, which can produce natural killer cells. In addition, a chemotherapeutic drug, an immunotherapeutic drug (Il-2 which enhances the production of CD8+ T cells) and an immunotherapy which directly increases the numbers of CD8+ T cells are administered at some rate. In the absence of any therapy or immune cells, the tumour grows logistically. It is killed by natural killer (NK) cells, by CD8+ T cells and by the chemotherapeutic drug. Chemotherapy also kills the immune cells.

In the absence of therapy, the authors perform a bifurcation analysis of the system, with the 
efficiency, $c$, of tumour cell kill by the NK cells as the bifurcation parameter. When the NK-kill rate is low, the high tumour equilibrium is the only stable steady state, whereas when the NK-kill rate is high, the zero tumour steady state is also stable and the final fate of the system (high tumour versus clearance) depends on the initial conditions. A second bifurcation analysis is performed with respect to the parameter $j$, which is the maximal rate at which the tumour recruits CD8+ $\mathrm{T}$ cells. For values of $j$ below a critical value, the tumour burden converges to a high value, corresponding to the only stable equilibrium. For values of $j$ above the critical value, the system is bistable and can converge to the zero tumour steady state or the high tumour burden steady state. The basin of attraction for tumour clearance has a low tumour initial tumour burden and non-negligible initial population of CD8+ T cells.

The authors compare numerical experiments to data from a mouse tumour. They estimate parameters and then apply chemotherapy, by allowing cytotoxic drug to be produced in simulations. For their chosen parameters, this fails to control the tumour. Similarly simulated immunotherapy, via an injection of CD8+ cells, also fails to control the tumour. When the two therapies are combined, however, the tumour is cleared. Similar numerical experiments are performed, estimating parameters for data from two human patients. The immune health of the patient (indicated by the initial numbers of CD8+ T cells, natural killer cells and circulating lymphocytes) affects the ability to clear the tumour. Immunotherapy can help to clear the tumour, but its effectiveness may be limited to clearing small tumours. In cases where chemotherapy or immunotherapy alone failed to clear the tumour, combined therapy could be effective. Patient-specific parameters (such as the immune-tumour lysis rates) could also affect the outcome of therapies. Finally vaccine therapy was simulated. Experimental data on mouse vaccine trials [84] showed that the vaccine increased $c$, the fractional tumour cell kill by the NK cells, $d$, the fractional tumour cell kill by the CD8+ $\mathrm{T}$ Cells, $g$, the maximal NK cell recruitment rate by the tumour and $j$, the maximum CD8+ $\mathrm{T}$ cell recruitment rate and decreased s, the steepness coefficient of the tumour-CD8+ T competition term. In combination with chemotherapy, this parameter-altering vaccine could limit the growth of a large tumour, provided it was administered sufficiently early in the tumour growth history.

Another model which addresses combination therapies is given in [85]. Here the idea is that tumour cells are exposed to a drug, which is a small-molecule inhibitor. Unfortunately, tumour cells can become resistant to the drug. The second therapy comes from infection with an oncolytic (i.e. specifically cancer cell killing) virus. Drug resistance has been shown commonly to confer (in the absence of the drug) a fitness advantage on tumour cells. Thus when drug-resistant and drugsensitive cancer tumour cells are infected with a virus, the faster growing drug-sensitive population may produce virus at such a rate as to overwhelm and eliminate the drug-resistant population. Sequential administration of the oncolytic virus and drug could then lead to complete elimination of the tumour cell population.

The model consists of ordinary differential equations to describe drug-sensitive and drugresistant tumour cell populations, infected or uninfected by the virus. Uninfected cells grow at rates which are thought to be lower, in the absence of the drug, if the tumour cells are drug-resistant. For example, in chronic myeloid leukaemia, imitinib-resistant mutant cells have been shown to have reduced growth rates. Uninfected cells become infected by interaction with infected cells at 
rates which are intermediate between mass action terms and frequency dependent terms (the latter being proportional to the frequency of infected cells rather than their density). Infected cells die at some rate. Provided that the rate of infection with the virus is similar or only slightly reduced in drug-resistant tumour cells, their lower growth rates, coupled with the high viral load produced by their drug-sensitive counterparts, can lead to extinction of the drug-resistant population. This process by which two populations do not directly compete, but the fitter one can drive the less fit population extinct if they are infected by the same pathogen, is termed 'apparent competition' in the population dynamics literature. If the virus grows fast enough to eliminate the drug- resistant tumour cells, the drug can then be administered to clear the drug-sensitive tumour cells. With the constant threat of mutation to give rise to new drug-resistant cells, the virus must be sufficiently efficient to clear these cells. The model is designed to be a proof of concept requiring experimental validation. In particular, chronic myeloid leukaemia, for which the best data on drug-resistant mutants and their relative fitnesses exists, has no known successful oncolytic virus. If the combined drug and virus treatments are shown to be effective then optimal scheduling will be an important area to explore.

Mathematical analysis of a class of models of the interaction between a single tumour cell population and an oncolytic virus are presented in [86]. The terms describing growth of the tumour and infection of tumour cells with virus are rather general and are categorised according to whether tumour clearance is possible.

Recent models [87], [88] address the spatiotemporal dynamics of a model of the response of tumour infiltrating cytotoxic lymphocytes (TICLs) to a small avascular, solid tumour with no necrotic core. TICLs include cytotoxic T lymphocytes, natural killer-like cells and lymphokineactivated killer cells. They are assumed to bind to tumour cells, forming complexes. These complexes can break up likely resulting either in survival of both cells or in the killing of the tumour cell, but with crucially very small probability, $p$, resulting in the inactivation of the TICL. These dynamics are termed the "kinetic scheme" and derive from the earlier work of [78], [79]. In addition, tumour cells grow at a logistic rate and TICLs proliferate at a saturating rate which increases with the density of tumour cell-TICL complexes. Both tumour cells and TICLs undergo random motion within a fixed domain with no flux boundaries, whilst random motion of the complexes is neglected. A diffusible chemokine is produced at a rate proportional to the density of complexes and it is assumed that TICLs can move up gradients of this by chemotaxis. In addition, TICLs are produced independently in part of the domain. Initially it is assumed that TICLs reside in that portion of the domain and tumour cells in the remainder.

On estimating the parameters, the authors find a regime in which there are moving peaks and troughs in the tumour cell density and TICL density throughout the spatial domain. When the cell densities are integrated across the whole domain to estimate total cell numbers, it is found that, at late times, the total number of tumour cells oscillates close to a steady value, corresponding to around 10 million cells. This is clearly subject to the accuracy of the parameter estimates. TICL levels also oscillate in this manner. The authors analyse the corresponding spatially homogeneous model (neglecting diffusion, chemotaxis and random migration) and find that, for their estimated parameter values, steady states of the model corresponding to tumour elimination and "tumour 
dormancy" (low tumour burden, high TICL) are unstable. There exists a stable limit cycle. The authors investigate the robustness of this limit cycle to changes in the parameters. They show that, if the rate of TICL binding to the tumour cells is below a threshold, the limit cycle disappears and dormant steady state is stable. If, on the contrary, $p$, the probability that a destructive complex break-up results in death of the tumour cell rather than inactivation of the TICL, is smaller than a threshold (0.9906 for the other estimated parameter values), then the limit cycle gives way to a stable "tumour invasion" state. In the spatial model, this results in travelling waves of tumour invasion.

Mathematical models have been used to describe the interaction between tumours and the innate and adaptive cellular immune system, the humoral immune system (leading to antibody production) and the influence of cytokines. The effects of various immunotherapies, including cytokine therapies, antibody therapies, adoptive cellular therapies, vaccinations and infection with oncolytic therapies have been studied.

\section{Discussion and Conclusions}

In breast cancer, patients who have undergone surgery to remove a primary tumour often suffer recurrences of the cancer sometimes at distant sites within the body, many years after surgery. Their tumours have been dormant in the body for those years. Typically patients die from such lategrowing metastases and not from the primary tumours. Therefore understanding the mechanisms by which tumours can remain dormant for extended periods is of great importance in the effort to reduce mortality from breast cancer. Many other cancers can also exhibit dormancy.

Some patients receive chemotherapy, hormonal therapy or radiotherapy in addition to surgery. This does not prevent much later recurrence of tumours. In fact, it appears that the significant effect of these therapies is to reduce early recurrence rates. It therefore seems plausible that dormant tumours are refractory to the therapies. There is additional evidence that many therapies act primarily on proliferating cells. Dormant tumours, in which cell proliferation is reduced, may thus escape therapeutic intervention. Clearly it is important to understand how dormancy may be controlled in order to try to i) make rapidly growing tumours dormant or ii) temporarily reduce dormancy during systemic therapies to increase their efficacy.

There are three main mechanisms that appear to contribute to dormant phases of tumour progression. These are i) nutrient limitation and in particular the limitations of an external blood supply prior to the angiogenic switch, ii) cellular quiescence and iii) immune responses to tumours. We have discussed each of these and in particular the relevant literature on mathematical modelling.

The literature on tumour angiogenesis and immune responses to tumours is huge and so only a partial review of these fields has been possible. A great deal of mathematical modelling has addressed both of these topics. An attempt has been made to describe a diverse selection of models from each area. The reader is referred to [3], [30], [20], [44], [80], [81] for further reading. Mathematical modelling can be useful in identifying the features of the modelled system which are most crucial in sustaining or limiting tumour growth. 
The structure of tumour vasculature can have important implications for the delivery of nutrients and drugs to the tumour. Therapies might try to modify the tumour vasculature in order to limit nutrient supply or increase drug access to tumours. More pertinently to a role in tumour dormancy, altering the morphology of the blood vessels near the tumour could affect the ability of cells to escape the tumour and metastasise. It has been shown that drugs can drastically modify the structure of tumour vasculature [89]. Mathematical modelling can help to predict key parameters of angiogenesis, vasculature or blood flow which affect drug supply, nutrient access or metastatic ability and may be targets for drugs.

The literature on mathematical models of solitary dormant cells is considerably less developed than that on tumour angiogenesis. Modelling cellular quiescence in cancer is clearly an important avenue for future work. Mathematical models like those described above can help to identify key components of the tumour cell cycle signalling pathway and inhibitors of cell cycle progression. In addition, of great importance will be models which attempt to explain the interactions between the tumour and its microenvironment, which lead to growth or dormancy. In particular, models which address the seeding of solitary dormant metastatic cells and their subsequent decision to grow into micrometastases may be of great importance in understanding and controlling metastatic disease. In addition, models, such as [67], which address the effect of cellular quiescence on tumour therapies are likely to be important. It is possible that cytotoxic therapies could be combined with therapies which temporarily awaken dormant tumours.

Immunotherapies are attractive as anti-tumour weapons, because they can specifically target tumour cells, and therefore have fewer side effects than chemotherapies or radiotherapies, and because they can be used to detect tumour cells as well as to destroy them. Mathematical modelling has addressed tumour immunotherapies for many years (see for example [80]). It can be very useful in understanding the often complex interactions between tumour cells and the various arms of the immune system. It can help to predict key parameters which determine whether the immune system can clear a tumour, whether the tumour will persist in a low-level dormant state or whether the tumour will grow in an uncontrolled fashion. It can also help to understand the influence of outside interventions, for example comparing the benefits of adoptive cellular therapies, cytokine therapies or combined therapies. In addition, mathematical modelling can be used as a test bed for new ideas, such as using viruses to eliminate drug resistant cancer cells [85].

Overall, it is clear that tumour dormancy is an important component of the natural history of cancer with implications for mortality and for therapy. Mathematical modelling has contributed and is very likely to contribute further to the understanding of tumour dormancy. Some of the key challenges which remain include understanding better the maintenance of solitary dormant cells, in particular what makes them refractory to systemic therapies and how the metastatic microenvironment influences the cell's decision to proliferate or become quiescent. In addition, in the realm of tumour immunotherapy, it will be important to understand immuno-editing, that is how the tumour evolves under immune attack, leading to escape from immune control. Lastly, it will be very important to understand more completely how the primary tumour can suppress proliferation and angiogenesis in metastases and whether these suppressive effects can be mimicked by external therapies. 


\section{Acknowledgements}

The author is grateful to the Leverhulme Trust for a fellowship which partly supported this work.

\section{References}

[1] C.A. Klein, D. Hoelzel. Systemic cancer progression and tumor dormancy: mathematical models meet single cell genomics. Cell Cycle, 5 (2006), No. 16, 1788-1798.

[2] R.A. Willis. The Spread of Tumors in the Human Body. Butterworth and Co. Ltd., London, 1952.

[3] J.A. Aguirre-Ghiso. Models, mechanisms and clinical evidence for cancer dormancy. Nature Rev. Cancer, 7 (2007), No. 11, 834-846.

[4] T.G. Karrison, D.J. Ferguson, P. Meier. Dormancy of Mammary Carcinoma after Mastectomy. J. Natl. Cancer Inst., 91 (1999), No. 1, 80-85.

[5] D. Weckermann, P. Mueller, F. Wawroschek, R. Harzmann, G. Riethmueller, G. Schlimok. Disseminated Cytokeratin Positive Tumour Cells in the Bone Marrow of Patients with Prostate Cancer: Detection and Prognostic value. J. Urol., 166 (2001), No. 2, 699-703.

[6] Early Breast Cancer Trialists' Collaborative Group (EBCTCG). Effects of chemotherapy and hormonal therapy for early breast cancer on recurrence and 15-year survival: an overview of the randomised trials. Lancet, 365 (2005), No. 9472, 1687-1717.

[7] T. Saphner, D.C. Tormey, R. Gray. Annual hazard rates of recurrence for breast cancer after primary therapy. J. Clin. Oncol., 14 (1996), No. 10, 2738-2746.

[8] L.E. Rutqvist, A. Wallgren, B. Nilsson. Is breast cancer a curable disease? A study of 14,731 women with breast cancer from the cancer registry of Norway. Cancer, 53 (1984), No. 8, 1793-1800.

[9] S. Meng, D. Tripathy, E.P. Frenkel, S. Shete, E.Z. Naftalis, J.F. Huth, P.D. Beitsch, M. Leitch, S. Hoover, D. Euhus, B. Haley, L. Morrison, T.P. Fleming, D. Herlyn, L.W.M.M. Terstappen, T. Fehm, T.F. Tucker, N. Lane, J. Wang, J.W. Uhr. Circulating tumour cells in patients with breast cancer dormancy. Clin. Cancer Res., 10, (2004), No. 24, 8152-8162.

[10] L. Norton, R. Simon, H.D. Brereton, A.E. Bogden. Predicting the course of Gompertzian growth. Nature, 264 (1976), No. 5586, 542-545.

[11] M.W. Retsky, R. Demicheli, D.E. Swartzendruber, P.D. Bame, R.H. Wardwell, G. Bonadonna, J.F. Speer, P. Valagussa. Computer Simulation of a breast cancer metastasis model. Breast Cancer Res. and Treat., 45 (1997), No. 2, 193-202. 
[12] H.J.G. Bloom, W.W. Richardson and E.J. Harries. Natural history of untreated breast cancer (1805-1933). Br. Med. J., 2 (1962), No. 5299, 213-221.

[13] S.E. Clare, F. Nakhlis, J.C. Panetta. Molecular biology of breast cancer metastasis: the use of mathematical models to determine relapse and to predict response to chemotherapy in breast cancer. Breast Cancer Res., 2 (2000), No. 6, 430-435.

[14] R. Demicheli, A. Abbatista, R. Micheli, P. Valagussa, G. Bonadonna. Time distribution of the recurrence risk for breast cancer patients undergoing mastectomy: further support about the concept of tumor dormancy. Breast Cancer Res. Treat., 41 (1996), No. 2, 177-185.

[15] R. Demicheli, R. Micheli, P. Valagussa, G. Bonadonna. re: Dormancy of mammary carcinoma after mastectomy. J. Natl. Cancer Inst., 92 (1999), No. 4, 347-348.

[16] R. Demicheli. Tumour dormancy: findings and hypotheses from clinical research on breast cancer. Semin. Cancer Biol., 11 (2001), No. 4, 297-305.

[17] T.G. Karrison, D.J. Ferguson, P. Meier. RESPONSE: re: Dormancy of mammary carcinoma after mastectomy., J. Natl. Cancer Inst., 92 (1999), No. 4, 348.

[18] M. Brackstone, J.L. Townson, A.F. Chambers. Tumour dormancy in breast cancer: an update. Breast Cancer Res., 9 (2007), No. 3, 208.

[19] R.P. Araujo and D.L.S. McElwain. A history of the study of solid tumour growth: the contribution of mathematical modelling. Bull. Math. Biol., 66 (2004), No. 5, 1039-1091.

[20] N. Bellomo, N.K. Li, P.K. Maini. On the foundations of cancer modelling: selected topics, speculations and perspectives. Math. Models Methods Appl. Sci., 18 (2008), No. 4, 593-646.

[21] J. Folkman. Tumor angiogenesis: therapeutic implications. N. Eng J. Med., 285 (1971), No. 21, 1182-1186.

[22] J. Folkman. Angiogenesis in cancer, vascular, rheumatoid and other diseases. Nature Med., 1 (1995), No. 1, 27-31.

[23] L. Holmgren, M.S. O'Reilly, J. Folkman. Dormancy of micrometastases: Balanced proliferation and apoptosis in the presence of angiogenesis suppression. Nature Med., 1 (1995), No. 2, 149-153.

[24] M.S. O’Reilly, L. Holmgren, Y. Shing, C. Chen, R.A. Rosenthal, M. Moses, W.S. Lane, Y. Cao, E.H. Sage, J. Folkman. Angiostatin: a novel angiogenesis inhibitor that mediates the suppression of metastases by a Lewis lung carcinoma. Cell 79 (1994), No. 2, 315-328.

[25] D. Hanahan, J. Folkman. Patterns and emerging mechanisms of the angiogenic switch during tumorigenesis. Cell, 86 (1996), No. 3, 353-364. 
[26] W. Risau, H. Sariola, H.-G. Zerwes, J. Sasse, P. Ekblom, R. Kemler, T. Doetschmann. Vasculogenesis and angiogenesis in embryonic-stem-cell-derived embryoid bodies. Development, 102 (1988), No. 3, 471-478.

[27] W. Risau. Mechansims of angiogenesis. Nature, 386 (1997), No. 6626, 671-674.

[28] M.F. Bolontrade, R.R. Zhou, E.S. Kleinerman. Vasculogenesis plays a role in the growth of Ewing's sarcoma in vivo. Clin. Cancer Res., 8 (2002), No. 11, 3622-3627.

[29] D. Ribatti, A. Vacca, F. Dammacco. New non-angiogenesis dependent pathways of tumour growth. Eur. J. Cancer, 39 (2003), No. 13, 1835-1841.

[30] N.V. Mantzaris, S. Webb, H.G. Othmer. Mathematical modeling of tumor-induced angiogenesis. J. Math. Biol., 49 (2004), No. 2, 111-187.

[31] M.A.J. Chaplain, S.R. McDougall, A.R.A. Anderson. Mathematical modeling of tumorinduced angiogenesis. Annu. Rev. Biomed. Eng., 8 (2006), 233-257.

[32] M. Baum, M.A.J. Chaplain, A.R.A. Anderson, M. Douek, J.S. Vaidya. Does breast cancer exist in a state of chaos?, Europ. J. Cancer, 35 (1999), No. 6, 886-891.

[33] A.R.A Anderson, M.A.J. Chaplain. Continuous and discrete models mathematical models of tumor-induced angiogenesis. Bull. Math. Biol., 60 (1998), No. 5, 857-899.

[34] V.R. Muthukkaruppan, L. Kubai, R. Auerbach. Tumor-induced neovascularization in the mouse eye. J. Natl. Cancer Inst., 69 (1982), No. 3, 699-705.

[35] T. Alarcon, H.M. Byrne, P.K. Maini. A cellular automaton model for tumour growth in inhomogeneous environment. J. Theor. Biol., 225 (2003), No. 2, 257-274.

[36] M.R. Owen, T. Alarcon, P.K. Maini, H.M. Byrne. Angiogenesis and vascular remodelling in normal and cancerous tissues. J. Math. Biol., 58 (2009), No.s 4-5, 689-721.

[37] S.R. McDougall, A.R.A. Anderson, M.A.J. Chaplain, J.A. Sherratt. Mathematical modelling of flow through vascular networks: implications for tumour-induced angiogenesis and chemotherapy strategies. Bull. Math. Biol., 64 (2002), No. 4, 673-702.

[38] M. Welter, K. Bartha, H. Rieger. Emergent vascular network inhomogeneities and resulting blood flow patterns in a growing tumor. J. Theor. Biol., 250 (2008), No. 2, 257-280.

[39] A.R. Pries, T.W. Secomb, P. Gaehtgens. Biophysical aspects of blood flow in the microvasculature. Cardiovsacular Research, 32 (1996), No. 4, 654-667.

[40] A.R. Pries, T.W. Secomb, P. Gaehtgens. Structural adaptation and stability of microvascular networks: theory and simulations. Am. J. Physiol. Heart Circ. Physiol., 275 (1998), No. 2, H349-H360. 
[41] A.R. Pries, B. Reglin, T.W. Secomb. Structural adaptation of microvascular networks: functional roles of adaptive responses. Am. J. Physiol. Heart Circ. Physiol., 281 (2001), No. 3, H1015-H1025.

[42] H.V. Jain, J.E. Noer, T.L. Jackson. Modeling the VEGF-Bcl-2-CXCL8 pathway in intratumoral angiogenesis. Bull. Math. Biol., 70 (2008), No. 1, 89-117.

[43] D. Wodarz, Y. Iwasa, N.L. Komarova. On the emergence of multifocal cancers. J. Carcinogenesis, 3 (2004), 13.

[44] D. Wodarz, N.L. Komarova. Computational biology of cancer: lecture notes and mathematical modeling. World Scientific Publishing, Singapore, 2005.

[45] S. Ramanujan, G.C. Koenig, T.P. Padera, B.R. Stoll, R.K. Jain. Local imbalance of proangiogenic and antiangiogenic factors: a potential mechanism of focal necrosis and dormancy in tumors. Cancer Research, 60 (2000), No. 5, 1442-1448.

[46] D. Wodarz, D.C. Krakauer. Genetic instability and the evolution of angiogenic tumor cell lines. Oncology Reports, 8 (2001), No. 6, 1195-1201.

[47] M.J. Plank, B.D. Sleeman, P.F. Jones. A Mathematical Model of Tumour Angiogenesis, Regulated by Vascular Endothelial Growth Factor and the Angiopoietins. J. Theor. Biol., 229 (2004), No. 4, 435-454.

[48] H.G. Othmer, A. Stevens. Aggregation, blowup, and collapse: the ABC's of taxis in reinforced random walks. SIAM J. Appl. Math., 57 (1997), No. 4, 1044-1081.

[49] G.N. Naumov, E. Bender, D. Zurakowski, S.-Y. Kand, D. Sampson, E. Flynn, R.S. Watnick, O. Straume, L.A. Akslen, J. Folkman, N. Almog. A model of human tumor dormancy: an angiogenic switch from the nonangiogenic phenotype. J. Natl. Cancer Inst., 98 (2006), No. 5, $316-325$.

[50] G. Bergers, L.E. Benjamin. Tumorigenesis and the angiogenic switch. Nature Rev. Cancer, 3 (2002), No. 6, 401-410.

[51] A. Abdollahi, C. Schwager, J. Kleeff, I. Esposito, S. Domhan, P. Peschke, K. Hauser, P. Hahnfelt, L. Hlatky, J. Debus, J.M. Peters, H. Friess, J. Folkman, P.E. Huber. Transcriptional network governing the angiogenic switch in human pancreatic cancer. PNAS, 104 (2007), No. 21, 12890-12895.

[52] P.T. Logan, B.F. Fernandes, S. Di Cesare, J.-C.A. Marshall, S.C. Maloney, M.N. Burnier. Single-cell tumor dormancy model of uveal melanoma. Clin. Exp. Metastasis, 25 (2008), No. 5, 509-516.

[53] J.L. Townson, A.F. Chambers. Dormancy of solitary metastatic cells. Cell Cycle, 5 (2006), No. $16,1744-1750$. 
[54] G.N. Naumov, I.C. MacDonald, P.M. Weinmeister, N. Kerkvliet, K.V. Nadkarni, S.M. Wilson, V.L. Morris, A.C. Groom, A.F. Chambers. Persistence of solitary mammary carcinoma cells in a secondary site: a possible contributor to dormancy. Cancer Res., 62 (2002), No. 7 , 2162-2168.

[55] J.A. Aguirre-Ghiso, D. Liu, A. Mignatti, K. Kovalski, L. Ossowaki. Urokinase receptor and fibronectin regulate the $E R K(M A P K)$ to $\mathrm{p} 38(M A P K)$ activity ratios that determine carcinoma cell proliferation or dormancy in vivo. Mol. Biol. Cell, 12 (2001), No. 4, 863-879.

[56] C.M. Shachaf, A.M. Kopelman, C. Arvanitis, . Karlsson, S. Beer, S. Mandl, M.H. Bachmann, A.D. Borowsky, B. Ruebner, R.D. Cardiff, Q. Yang, J.M. Bishop, C.H. Contag, D.W. Felsher. MYC inactivation uncovers pluripotent differentiation and tumour dormancy in hepatocellular carcinoma. Nature, 431 (2004), No. 7012, 1112-1117.

[57] M. Guba, G. Cernaianu, G. Koehl, E.K. Geissler, K.-W. Jauch, M. Anthuber, W. Falk, M. Steinbauer. A primary tumor promotes dormancy of solitary tumor cells before inhibiting angiogenesis. Cancer Res., 61 (2001), No. 14, 5575-5579.

[58] A.L. Allan, S.A. Vantyghem, A.B. Tuck, A.F. Chambers. Tumor dormancy and cancer stem cells: implications for the biology and treatment of breast cancer metastasis. Breast Disease, 26 (2006, 2007), No. 1, 87-98.

[59] M. Balic, H. Lin, L. Young, D. Hawes, A. Giuliano, G. McNamara, R.H. Datar, R.J. Cote. Most early disseminated cancer cells detected in bone marrow of breast cancer patients have a putative stem cell phenotype. Clin. Cancer Res., 12 (2006), No. 19, 5615-5621.

[60] T. Alarcon, R. Marches, K.M. Page. Mathematical models of the fate of lymphoma B cells after antigen receptor ligation with specific antibodies. J. Theor. Biol., 240 (2006), No. 1, 54-71.

[61] T. Alarcon, H.M. Byrne, P.K. Maini. Towards whole organ modelling of tumour growth. Prog. Biophys. Mol. Biol. 85 (2004), No.s 2-3, 451-472.

[62] T. Alarcon, H.M. Byrne, P.K. Maini. A multiple scale model for tumor growth. Multiscale Model. Simul., 3 (2005), No. 2, 440-475.

[63] H.M. Byrne, M.R. Owen, T. Alarcon, J. Murphy, P.K. Maini. Modelling the response of vascular tumours to chemotherapy. Math. Mod. Meth. Appl. Sci., 16 (2006), No. 7S, 12191241.

[64] B. Ribba, T. Colin, S. Schnell. A mathematical model of cancer and its use in analyzing irradiation therapies. Theor. Biol. Med. Model., 3 (2006), 7.

[65] V. Hatzimanikatis, K.H. Lee, J.E. Bailey. A mathematical description of refulation of the G1-S transition of the mammalian cell cycle. Biotechnol. bioeng., 65 (1999), No. 6, 631-637. 
[66] M. Gyllenberg. G.F. Webb. Quiescence as an explanation of Gompertzian tumor growth. Growth, dev. aging, 86 (1987), No.s 1-2, 67-95.

[67] N.L. Komarova, D. Wodarz. Effect of cellular quiescence on the success of targeted CML therapy. PLoS ONE, 2 (2007), No. 10, e990.

[68] J.W. Uhr, R.H. Scheuermann, N.E. Street, E.S. Vitetta. Cancer dormancy: opportunities for new therapeutic approaches. Nature Med., 3 (1997), No. 5, 505-509.

[69] B. Quesnel. Dormant tumor cells as a therapeutic target? Cancer Lett., 267 (2008), No. 1, $10-17$.

[70] G.P. Dunn, A.T. Bruce, H. Ikeda, L.J. Old, R.D. Schreiber. Cancer immunoediting: from immunosurveillance to tumor escape. Nature Immunology, 3 (2002), No. 11, 991-999.

[71] K.J. Weinhold, L.T. Goldstein, E.F. Wheelock. Tumour-dormant states established with L5178Y lymphoma cells in immunised syngeneic murine hosts. Nature, 270 (1977), No. 5632, $59-61$.

[72] H. Siu, E.S. Vitetta, R.D. May, J.W. Uhr. Tumor dormancy. I. Regression of BCL1 tumor and induction of a dormant tumor state in mice chimeric at the major histocompatibility complex. J. Immunol., 137 (1986), No. 4, 1376-1382.

[73] C.G. Clemente, M.C. Mihm Jr., R. Bufalino, S. Zurrida, P. Collini, N. Cascinelli. Prognostic value of tumor infiltrating lymphocytes in the vertical growth phase of primary cutaneous melanoma. Cancer, 77 (1996), No. 7, 1303-1310.

[74] J. Galon, A. Costes, F. Sanchez-Cabo, A. Kirilovsky, B. Mlecnik, C. Lagorce-Pags, M. Tosolini, M. Camus, A. Berger, P. Wind, F. Zinzindohou, P. Bruneval, P.-H. Cugnenc, Z. Trajanoski, W.-H. Fridman, F. Pags. Type, density and location of immune cells within human colorectal tumors predict clinical outcome. Science, 313 (2006), No. 5795, 1960-1964.

[75] E. Sato, S. H. Olson, J. Ahn, B. Bundy, H. Nishikawa, F. Qian, A.A. Jungbluth, D. Frosina, S. Gnjatic, C. Ambrosone, J. Kepner, T. Odunsi, G. Ritter, S. Lele, Y.-T. Chen, H. Ohtani, L.J. Old, K. Odunsi. Intraepithelial CD8+ tumor-infiltrating lymphocytes and a high CD8+/regulatory $T$ cell ratio are associated with favorable prognosis in ovarian cancer. Proc. Natl. Acad. Sci., 102 (2005), No. 51, 18538-18543.

[76] C.M. Koebel, W. Vermi, J.B. Swann, N. Zerafa, S.J. Rodig, L.J. Old, M.J. Smyth, R.D. Schreiber. Adaptive immunity maintains occult cancer in an equilibrium state. Nature, 450 (2007), No. 7171, 903-907.

[77] K.M. Page, J.W. Uhr. Mathematical models of cancer dormancy. Leukemia and Lymphoma, 46 (2005), No. 3, 313-327. 
[78] V.A. Kuznetsov, I.A. Makalkin, M.A. Taylor, S. Perelson. Nonlinear dynamics of immunogenic tumors: parameter estimation and global bifurcation analysis. Bull. Math. Biol., 56 (1994), No. 2, 295-321.

[79] V.A. Kuznetsov, G.D. Knott. Modeling tumor regrowth and immunotherapy. Math. Comp. Modelling, 33 (2001), No.s 12-13, 1275-1287.

[80] J.A. Adam, N. Bellomo. A survey of tumor-immune system dynamics (modeling and simulation in science, engineering and technology). Birkhaeuser, Boston, 1996.

[81] N. Bellomo, L. Preziosi. Modelling and mathematical problems related to tumor evolution and its interaction with the immune system. Math. Comp. Model., 32 (2000), No.s 3-4, 413452.

[82] D. Kirschner, J.C. Panetta. Modeling immunotherapy of the tumor-immune interaction. J. Math. Biol., 37 (1998), No. 3, 235-252.

[83] L.G. De Pillis, W. Gu, A.E. Radunskaya. Mixed immunotherapy and chemotherapy of tumors: modeling, applications and biological interpretations. J. Theor. Biol., 238 (2006), No. 4, 841-862.

[84] A. Diefenbach, E.R. Jensen, A.M. Jamison, D. Raulet. Rael and H60 ligands of the NKG2D receptor stimulate tumor immunity. Nature, 413 (2001), No. 6852, 165-171.

[85] D. Wodarz. Use of oncolytic viruses for the eradication of drug-resistant cancer cells. J. R. Soc. Interface, 6 (2009), No. 31, 179-186.

[86] D. Wodarz, N. Komarova. Towards predictive computational models of oncolytic virus therapy: basis for experimental validation and model selection. PLoS One, 4 (2009), No. 1, e4271.

[87] A. Matzavinos, M.A.J. Chaplain, V.A. Kuznetsov. Mathematical modelling of the spatiotemporal response of cytotoxic T-lymphocytes to a solid tumour. Math. Medicine and Biology: A Journal of the IMA, 21 (2004), No. 1, 1-34.

[88] A. Matzavinos. Dynamic irregular patterns and invasive wavefronts: the control of tumour growth by cytotoxic T-lymphcytes. In: Selected topics in cancer modeling (modeling and simulation in science engineering and technology), Birkhauser, Boston, 2008.

[89] A.W. Le Serve, K. Hellman. Metastases and the normalization of tumour blood vessels by ICRF 159: a new type of drug action. Br. Med. J., 1 (1972), No. 5800, 597-601. 